\title{
Protection of Port and Yard Equipment's and Back to Operational Effectiveness under Abnormal Cyclonic Conditions
}

\author{
G .Vara Prasad Babu, M. Pramila Devi
}

\begin{abstract}
The purpose of this study is to assess the effect of the cyclones and its influence on port performance. The techniques of percentages and frequencies are applied to 141 cyclones as a sample, obtained from various cyclones happened around the world. The results identify and measure the factors that characterize the operational factors and affects port performance. Factors effecting including service level, partner network, ship services, cargo services, logistics services and advanced services, affect port performance, operational performance, effectiveness and efficiency. The primary contribution of this study deals with analysing the criticality of the cyclone and taking the appropriate measures, considering wind speeds ranging from 20 to $350 \mathrm{~km} / \mathrm{h}$. PYE (Port and Yard Equipment) which sub part of the equipment should be locked on safety measures and are more likely to get effected by the wind speeds and the sub equipment parts need to belocking considered are long travel, slew and boom conveyor.
\end{abstract}

Keywords: port and yard Equipment's, stacker cum reclaimers, long travel, slew, boom Conveyor.

\section{INTRODUCTION}

According to Indian meteorological department, ministry of earth science, government of India, the cyclone warnings are issued to state government officials in four stages. The First Stage warning known as "PRECYCLONE WATCH" issued 72 hours in advance contains early warning about the development of a cyclonic disturbance. The Second Stage warning known as "CYCLONE ALERT" is issued at least $48 \mathrm{hrs}$. in advance of the expected commencement of adverse weather over the coastal areas. The Third Stage warning known as "CYCLONE WARNING" issued at least 24 hours in advance of the expected commencement of adverse weather over the coastal areas. The Fourth Stage of warning known as "POST LANDFALL OUTLOOK" is issued by the concerned ACWCs/CWCs/and CWD at HQ at least 12 hours in advance of expected time of landfall.It gives likely direction of movement of the cyclone after its landfall and adverse weather likely to be experienced in the interior areas.

Revised Manuscript Received on February 05, 2020.

* Correspondence Author

G. Vara Prasad Babu*, Department of Mechanical Engineering, Andhra University, Visakhapatnam, Andhra Pradesh,India. Email: vara2637@gmail.com

Prof. M. Pramila Devi, Department of Mechanical Engineering, Andhra University, Visakhapatnam, Andhra Pradesh, India. Email: pramiladevi_m@yahoo.co.in

(c) The Authors. Published by Blue Eyes Intelligence Engineering and Sciences Publication (BEIESP). This is an open access article under the CC BY-NC-ND license (http://creativecommons.org/licenses/by-nc-nd/4.0/)

The equipment's considered are

1. Ship unloaders

2. Ship loaders

3. Harbour mobile cranes

4. Stacker

5. Reclaimers

6. Stacker cum reclaimers

7. Wagon loader

Under abnormal cyclone conditions the storm anchoring locking devices kept ready in all aspects of equipment safety by means of safe parking positions including buffer end stopper locking, the evaluating criteria of how sever the wind speed will be the locking practices can be evaluated by which the respective equipment could be brought back in to operational effectively with in the minimum down time.

To minimize the sum of job waiting times, $\mathrm{A}$ branch and bound algorithm is proposed to solve the scheduling problem optimally [1]. The most important objective for a port container terminal is to increase its throughput or, in particular, to decrease the turnaround times of ships [2]. The major port operations and minimize the various delays [3]. The turnaround time of a ship depends on the effectiveness of allocating and scheduling key resources, such as berths, yards, quay cranes, yard cranes and trucks [4]. The problems faced by the Indian ports today[5].The average turnaround time could be cut down by $50 \%$, India's manufacturing exports may increase by at least $20-25 \%$. [6]

\section{METHODOLOGY}

A Different Cyclones around the World with Wind Speeds:

Studying different cyclones with varying wind speeds all the three sub parts of the equipment need not to be locked, depends on thecriticality of wind speeds locking arrangement can be considered to bring back to operational effectiveness

Considering the wind speeds with the interval of

The wind speeds taken from 20-110 being assigned as 1

The wind speeds taken from 110-200 being assigned as 2

The wind speeds taken from 200-350 being assigned as 3

With the class intervals respectively.

The following list is subdivided by basins. Data listed are provided by the official Regional Specialized Meteorological Centre,

1. North Atlantic Ocean

2. Eastern Pacific Ocean

3. Western North Pacific Ocean

4. North Indian Ocean 
Protection of Port and Yard Equipment's and Back to Operational Effectiveness under Abnormal Cyclonic Conditions

5. South-West Indian Ocean

6. Australian Region

7. South Pacific Ocean

8. South Atlantic Ocean

Table no.1: various cyclones with their names and occurrence of the year and wind speeds

\begin{tabular}{|c|c|c|c|}
\hline S.No & Cyclone & Year & $\begin{array}{l}\text { Wind } \\
\text { Speed }\end{array}$ \\
\hline 1 & South Atlantic & 1974 & 45 \\
\hline 2 & Bapo & 2015 & 65 \\
\hline 3 & Cari & 2015 & 65 \\
\hline 4 & Deni & 2016 & 75 \\
\hline 5 & Anita & 2010 & 85 \\
\hline 6 & Arani & 2011 & 85 \\
\hline 7 & Eçaí & 2016 & 100 \\
\hline 8 & Catarina & 2004 & 155 \\
\hline 9 & Bonita & 1995-96 & 180 \\
\hline 10 & Marlene & 1994-95 & 180 \\
\hline 11 & Hudhud & 2014 & 185 \\
\hline 12 & Daniella & 1996-97 & 190 \\
\hline 13 & Litanne & 1993-94 & 190 \\
\hline 14 & Pakistan & 1999 & 195 \\
\hline 15 & Floyd & 2005-06 & 195 \\
\hline 16 & Giri & 2010 & 195 \\
\hline 17 & Two & 1963 & 195 \\
\hline S.No & Cyclone & Year & $\begin{array}{l}\text { Wind } \\
\text { Speed }\end{array}$ \\
\hline 18 & Geralda & 1993-94 & 200 \\
\hline 19 & Theodore & 1993-94 & 200 \\
\hline 20 & Andhra Pradesh & 1977 & 205 \\
\hline 21 & Beni & $2002-03$ & 205 \\
\hline 22 & Betty & 1987 & 205 \\
\hline 23 & Carina & 2005-06 & 205 \\
\hline 24 & Dovi & $2002-03$ & 205 \\
\hline 25 & Forrest & 1983 & 205 \\
\hline 26 & Fran & 1991-92 & 205 \\
\hline 27 & Gay & 1992 & 205 \\
\hline 28 & George & 2006-07 & 205 \\
\hline 29 & Glenda & 2005-06 & 205 \\
\hline 30 & Guillaume & 2001-02 & 205 \\
\hline 31 & Holly & 1987 & 205 \\
\hline 32 & Hope & 1979 & 205 \\
\hline 33 & Mahina & 1899 & 205 \\
\hline 34 & Marge & 1983 & 205 \\
\hline 35 & Nepartak & 2016 & 205 \\
\hline 36 & Nilofar & 2014 & 205 \\
\hline 37 & Oscar & 1982-83 & 205 \\
\hline 38 & Peggy & 1986 & 205 \\
\hline 39 & Sanba & 2012 & 205 \\
\hline 40 & Zeb & 1998 & 205 \\
\hline 41 & Chris-Damia & 1981-82 & 210 \\
\hline 42 & BOB 02 & 1994 & 215 \\
\hline 43 & India & 2001 & 215 \\
\hline 44 & Bento & 2004-05 & 215 \\
\hline 45 & Chapala & 2015 & 215 \\
\hline 46 & Dina & $2001-02$ & 215 \\
\hline 47 & Erica & $2002-03$ & 215 \\
\hline 48 & Fay & 2003-04 & 215 \\
\hline 49 & Haima & 2016 & 215 \\
\hline 50 & Heta & 2003-04 & 215 \\
\hline 51 & Hondo & $2007-08$ & 215 \\
\hline 52 & Kalunde & 2002-03 & 215 \\
\hline 53 & Meena & 2004-05 & 215 \\
\hline 54 & Olaf & 2004-05 & 215 \\
\hline
\end{tabular}

\begin{tabular}{|c|c|c|c|}
\hline 55 & Phailin & 2013 & 215 \\
\hline 56 & Ruth & 1991 & 215 \\
\hline 57 & Sidr & 2007 & 215 \\
\hline 58 & Soudelor & 2015 & 215 \\
\hline 59 & Ului & 2009-10 & 215 \\
\hline 60 & Vance & 1998-99 & 215 \\
\hline 61 & Vongfong & 2014 & 215 \\
\hline 62 & Abby & 1983 & 220 \\
\hline S.No & Cyclone & Year & $\begin{array}{l}\text { Wind } \\
\text { Speed }\end{array}$ \\
\hline 63 & Adeline-Juliet & 2004-05 & 220 \\
\hline 64 & Bansi & 2014-15 & 220 \\
\hline 65 & Bruce & $2013-14$ & 220 \\
\hline 66 & Dot & 1985 & 220 \\
\hline 67 & Edzani & 2009-10 & 220 \\
\hline 68 & Elsie & 1981 & 220 \\
\hline 69 & Flo & 1990 & 220 \\
\hline 70 & Gwenda & 1998-99 & 220 \\
\hline 71 & Hary & $2001-02$ & 220 \\
\hline 72 & Hina & 1984-85 & 220 \\
\hline 73 & Hudah & $\begin{array}{l}1999- \\
2000\end{array}$ & 220 \\
\hline 74 & Mac & 1982 & 220 \\
\hline 75 & Meranti & 2016 & 220 \\
\hline 76 & Odile & 2014 & 220 \\
\hline 77 & Rita & 1978 & 220 \\
\hline 78 & Vanessa & 1984 & 220 \\
\hline 79 & Wynne & 1980 & 220 \\
\hline 80 & Yuri & 1991 & 220 \\
\hline 81 & Andhra Pradesh & 1990 & 230 \\
\hline 82 & Amy & 1979-80 & 230 \\
\hline 83 & Bess & 1982 & 230 \\
\hline 84 & Eunice & 2014-15 & 230 \\
\hline 85 & Gafilo & 2003-04 & 230 \\
\hline 86 & Gay & 1989 & 230 \\
\hline 87 & Gloria & 1985 & 230 \\
\hline 88 & Graham & 1991-92 & 230 \\
\hline 89 & Haiyan & 2013 & 230 \\
\hline 90 & Hellen & 2013-14 & 230 \\
\hline 91 & Joan & $1975-76$ & 230 \\
\hline 92 & Megi & 2010 & 230 \\
\hline 93 & Percy & 2004-05 & 230 \\
\hline 94 & Ron & 1997-98 & 230 \\
\hline 95 & Susan & 1997-98 & 230 \\
\hline 96 & Winston & 2015-16 & 230 \\
\hline 97 & Bangladesh & 1991 & 240 \\
\hline 98 & Gonu & 2007 & 240 \\
\hline 99 & Inigo & $2002-03$ & 240 \\
\hline 100 & Opal & 1995 & 240 \\
\hline 101 & Three & 1963 & 240 \\
\hline 102 & Zoe & 2002-03 & 240 \\
\hline 103 & Bahamas & 1929 & 250 \\
\hline 104 & Fantala & 2015-16 & 250 \\
\hline 105 & Floyd & 1999 & 250 \\
\hline 106 & Igor & 2010 & 250 \\
\hline S.No & Cyclone & Year & $\begin{array}{l}\text { Wind } \\
\text { Speed }\end{array}$ \\
\hline 107 & Monica & $2005-06$ & 250 \\
\hline 108 & Orson & $1988-89$ & 250 \\
\hline 109 & Pam & 2014-15 & 250 \\
\hline 110 & Paradip & 1999 & 260 \\
\hline
\end{tabular}

\section{Published By:}

Blue Eyes Intelligence Engineering \& Sciences Publication 


\begin{tabular}{|l|l|l|l|}
\hline 111 & Ava & 1973 & 260 \\
\hline 112 & Celia & 2010 & 260 \\
\hline 113 & Elida & 2002 & 260 \\
\hline 114 & Gilma & 1994 & 260 \\
\hline 115 & Guillermo & 1997 & 260 \\
\hline 116 & Hattie & 1961 & 260 \\
\hline 117 & Hernan & 2002 & 260 \\
\hline 118 & Hugo & 1989 & 260 \\
\hline 119 & Ioke & 2006 & 260 \\
\hline 120 & Marie & 2014 & 260 \\
\hline 121 & Tip & 1979 & 260 \\
\hline 122 & Cuba & 1924 & 270 \\
\hline 123 & Isabel & 2003 & 270 \\
\hline 124 & Ivan & 2004 & 270 \\
\hline 125 & Kenna & 2002 & 270 \\
\hline 126 & Cuba & 1932 & 280 \\
\hline 127 & Andrew & 1992 & 280 \\
\hline 128 & Camille & 1969 & 280 \\
\hline 129 & David & 1979 & 280 \\
\hline 130 & Dean & 2007 & 280 \\
\hline 131 & Janet & 1955 & 280 \\
\hline 132 & Katrina & 2005 & 280 \\
\hline 133 & Mitch & 1998 & 285 \\
\hline 134 & Rick & 2009 & 285 \\
\hline 135 & Rita & 2005 & 285 \\
\hline 136 & Labor Day & 1935 & 295 \\
\hline 137 & Gilbert & 1988 & 295 \\
\hline 138 & Linda & 1997 & 295 \\
\hline 139 & Wilma & 2005 & 295 \\
\hline 140 & Allen & 1980 & 305 \\
\hline 141 & Patricia & 2015 & 345 \\
\hline
\end{tabular}

Source:

Atlantic Hurricane Best Track File 1851-2016

East Pacific Hurricane Best Track File 1851-2016

Typhoon information for theWestern Pacific ocean

Tropical Cyclone Best Track Information for the North Indian Ocean 1851-2016[

"TCWC Wellington Best Track Data 1967-2006

"Second only south Atlantic tropical storm: 90Q, moving away from Brazil".

\section{B CATEGORZATION OF WIND SPEEDS}

The wind speeds ranges been segregated in to three categories given in Table no.2 and equipment sub parts locking being assigned for safety values to the labels under abnormal cyclonic conditions.

Table no.2: Categorization of wind speeds

\begin{tabular}{|l|l|c|}
\hline $\begin{array}{l}\text { Wind } \\
\text { Speed }\end{array}$ & \multicolumn{1}{|c|}{ Sub-parts (Safety Labels) } & $\begin{array}{l}\text { Safety } \\
\text { Values }\end{array}$ \\
\hline $20-110$ & long travel & 1 \\
\hline $110-200$ & long travel and slew & 2 \\
\hline $200-350$ & long travel, slew and boom conveyor & 3 \\
\hline
\end{tabular}

From the table no.1 data of cyclones with wind speeds being put in ascending order and assigned the values of wind speed ranges as below

Table no.3: Ascending order of wind speeds from table no.1

\begin{tabular}{|c|c|c|}
\hline S.No & Wind Speed & Safety \\
\hline 1 & 45 & 1 \\
\hline 2 & 65 & 1 \\
\hline
\end{tabular}

\begin{tabular}{|c|c|c|}
\hline 3 & 65 & 1 \\
\hline 4 & 75 & 1 \\
\hline 5 & 85 & 1 \\
\hline 6 & 85 & 1 \\
\hline 7 & 100 & 1 \\
\hline 8 & 155 & 2 \\
\hline 9 & 180 & 2 \\
\hline 10 & 180 & 2 \\
\hline 11 & 185 & 2 \\
\hline 12 & 190 & 2 \\
\hline 13 & 190 & 2 \\
\hline 14 & 195 & 2 \\
\hline 15 & 195 & 2 \\
\hline 16 & 195 & 2 \\
\hline 17 & 195 & 2 \\
\hline 18 & 200 & 2 \\
\hline 19 & 200 & 2 \\
\hline 20 & 205 & 3 \\
\hline 21 & 205 & 3 \\
\hline 22 & 205 & 3 \\
\hline 23 & 205 & 3 \\
\hline 24 & 205 & 3 \\
\hline 25 & 205 & 3 \\
\hline 26 & 205 & 3 \\
\hline S.No & Wind Speed & Safety \\
\hline 27 & 205 & 3 \\
\hline 28 & 205 & 3 \\
\hline 29 & 205 & 3 \\
\hline 30 & 205 & 3 \\
\hline 31 & 205 & 3 \\
\hline 32 & 205 & 3 \\
\hline 33 & 205 & 3 \\
\hline 34 & 205 & 3 \\
\hline 35 & 205 & 3 \\
\hline 36 & 205 & 3 \\
\hline 37 & 205 & 3 \\
\hline 38 & 205 & 3 \\
\hline 39 & 205 & 3 \\
\hline 40 & 205 & 3 \\
\hline 41 & 210 & 3 \\
\hline 42 & 215 & 3 \\
\hline 43 & 215 & 3 \\
\hline 44 & 215 & 3 \\
\hline 45 & 215 & 3 \\
\hline 46 & 215 & 3 \\
\hline 47 & 215 & 3 \\
\hline 48 & 215 & 3 \\
\hline 49 & 215 & 3 \\
\hline 50 & 215 & 3 \\
\hline 51 & 215 & 3 \\
\hline 52 & 215 & 3 \\
\hline 53 & 215 & 3 \\
\hline 54 & 215 & 3 \\
\hline 55 & 215 & 3 \\
\hline 56 & 215 & 3 \\
\hline 57 & 215 & 3 \\
\hline 58 & 215 & 3 \\
\hline 59 & 215 & 3 \\
\hline 60 & 215 & 3 \\
\hline
\end{tabular}




\begin{tabular}{|c|c|c|c|c|c|}
\hline 61 & 215 & 3 & 122 & 270 & 3 \\
\hline 62 & 220 & 3 & 123 & 270 & 3 \\
\hline 63 & 220 & 3 & 124 & 270 & 3 \\
\hline 64 & 220 & 3 & 125 & 270 & 3 \\
\hline 65 & 220 & 3 & 126 & 280 & 3 \\
\hline 66 & 220 & 3 & 127 & 280 & 3 \\
\hline 67 & 220 & 3 & 128 & 280 & 3 \\
\hline 68 & 220 & 3 & 129 & 280 & 3 \\
\hline 69 & 220 & 3 & 130 & 280 & 3 \\
\hline 70 & 220 & 3 & S.No & Wind Speed & Safety \\
\hline 71 & 220 & 3 & 131 & 280 & 3 \\
\hline 72 & 220 & 3 & 132 & 280 & 3 \\
\hline 73 & 220 & 3 & 133 & 285 & 3 \\
\hline 74 & 220 & 3 & 134 & 285 & 3 \\
\hline 75 & 220 & 3 & 135 & 285 & 3 \\
\hline 76 & 220 & 3 & 136 & 295 & 3 \\
\hline 77 & 220 & 3 & 137 & 295 & 3 \\
\hline 78 & 220 & 3 & 138 & 295 & 3 \\
\hline S.No & Wind Speed & Safety & 139 & 295 & 3 \\
\hline 79 & 220 & 3 & 140 & 305 & 3 \\
\hline 80 & 220 & 3 & 141 & 345 & 3 \\
\hline
\end{tabular}

Table no. 4: percentages and Frequency Table of equipment subparts locking.

\begin{tabular}{|l|c|c|}
\hline Safety & Occurrence & Cumulative \\
\hline Long Travel & $\begin{array}{c}7 \\
(5.0 \%)\end{array}$ & $\begin{array}{c}7 \\
(5.0 \%)\end{array}$ \\
\hline Slew Lock & $\begin{array}{c}12 \\
(8.5 \%)\end{array}$ \\
\hline Boom Conveyor & $\begin{array}{c}122 \\
(13.5 \%)\end{array}$ \\
\hline
\end{tabular}

Within the Parenthesis value indicates the safety percentages of equipment subparts.

At a class interval of 20 to $110 \mathrm{~km} / \mathrm{h}$ wind speeds along travel can be locked, simultaneously with the increase intensity of wind speed 110 to $200 \mathrm{~km} / \mathrm{hr}$ combination of long travel and slew can be locked, similarly in a progressive rapidly seemingly manner at 200 to $350 \mathrm{~km} / \mathrm{hr}$ component elements of individually distinct long travel, slew and boom conveyor can be locked together. Wise considering the three class intervals the re-operational effective turnaround time is drastically reduced by a shift time or so.

\section{Occurance}

\begin{tabular}{|l|l|l|}
\hline 106 & 250 & 3 \\
\hline 107 & 250 & 3 \\
\hline 108 & 250 & 3 \\
\hline 109 & 250 & 3 \\
\hline 110 & 260 & 3 \\
\hline 111 & 260 & 3 \\
\hline 112 & 260 & 3 \\
\hline 113 & 260 & 3 \\
\hline 114 & 260 & 3 \\
\hline 115 & 260 & 3 \\
\hline 116 & 260 & 3 \\
\hline 117 & 260 & 3 \\
\hline 118 & 260 & 3 \\
\hline 119 & 260 & 3 \\
\hline 120 & 260 & 3 \\
\hline 121 & 260 & 3 \\
\hline
\end{tabular}

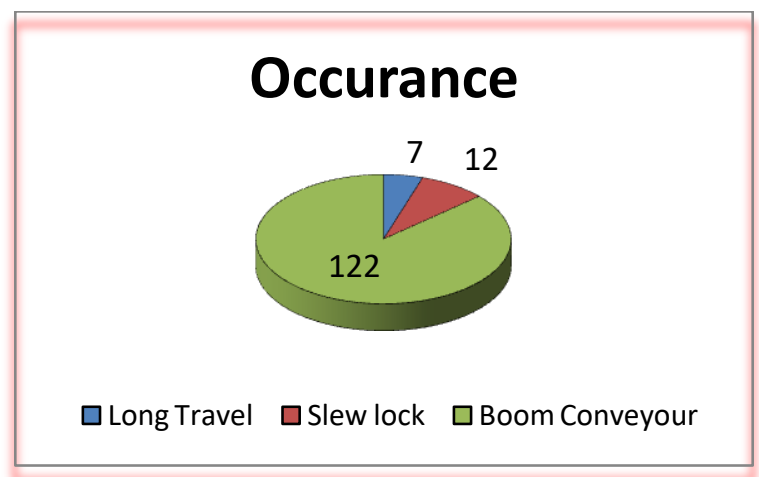

Fig. 1: Under abnormal cyclonic conditions locking of equipment sub parts at various wind speeds 
All the three sub parts of the equipment's mentioned need not to be locked with the cyclone alert the locking arrangements be considered with the criticality of wind speeds and the intensity of rain due slides of cargo can enter in to long travel boogies, hence with the alert of cyclones equipment's can be kept at parking positions and boom can be rested for equipment safety and other parameters be considered by studying the cyclone on dated of occurrence.

\section{RESULTS AND DISCUSSIONS}

1. For port and yard equipment's locking arrangements can be done by analysing the criticality of cyclones with their directions and wind speeds periodically considering the facts of operational hampering.

2. Initially Boom can be locked under cyclonic alerts, thereby minimizing down time and getting back the respective equipment's to operations, hence reducing turnaround time of the vessel under theses abnormal conditions also.

3. Boom conveyor locking helps the equipment in tact with in the parking premises, by which fouling with other super structure / vessel hatch considerations.

\section{CONCLUSION}

This study is made for protecting the port and yard equipment's by using various safety features and the equipment safety and getting back the equipment in to operational effectiveness with in the minimum turnaround time.

\section{ACKNOWLEDGEMENT}

I express my deep gratitude towards the management of Ganagavaram port limited for their valuable support and help in my work.

\section{REFERENCES}

1. W.C. Ng , K.L., Mak,"Yard crane scheduling in port container terminals", Applied Mathematical Modelling 29 Elsevier pp. 263276, 2005.

2. McKinsey and Company, Inc., Containerization: the key to low transport, a report by McKinsey and Company, Inc. for the British Transport Docks Board, 1967.

3. Saurabh Pratap, AshutoshNayak, AkhileshKumar, NaoufelCheikhrouhou and Manoj KumarTiwari, "An integrated decision support system for berth and ship unloader allocation in bulk material handling port”, Volume 106, pp. 386-399,elsevier 2017.

4. J. Bramel, D. Simchi-Levi, The Logic of Logistics: Theory, Algorithms, and Applications for LogisticsManagement, SpringerVerlag, New York, 1997.

5. DE MONIE, G."The problems faced by Indian ports today. Maritime Policy \& Management”,22(3), 235-238.1995

6. How operational efficiency of India's ports impacts its manufacturing exports

7. National Hurricane Centre; Hurricane Research Division (April 11, 2017). "Atlantic hurricane best track (HURDAT version 2)". United States National Oceanic and Atmospheric Administration.Retrieved May 26, 2017.

8. "Western North Pacific Typhoon best track file 1951-2017". Japan Meteorological Agency. 2010-01-13. Retrieved 2010-01-13.

9. Jump up Regional Specialised Meteorological Centre (RSMC) Tropical Cyclones, New Delhi. "IMD Best track data 1990-2015". India Meteorological Department.

10. Four Stage Warning".India Meteorological Department.Retrieved 23 October 2016.

\section{AUTHOR PROFILE:}

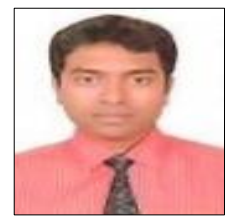

G. Vara Prasad Babu He is working as Deputy Manager at Gangavaram Port Limited, Visakhapatnam and Currently pursuing $\mathrm{PhD}$. in the department of mechanical engineering, Andhra University, Visakhapatnam, Andhra Pradesh, India. Received Master of Engineering (Industrial Engineering) and MBA (HRM) In Andhra University, Visakhapatnam, Andhra Pradesh, India.

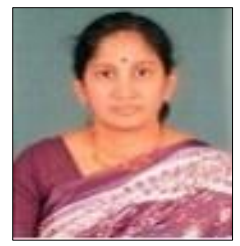

Prof. M. Pramila Devi received Ph.D. in the department of mechanical engineering, Andhra University, Visakhapatnam, Andhra Pradesh, India. She is working as a professor Andhra university, Visakhapatnam, Andhra Pradesh, India. 